ORIGINAL ARTICLE

\title{
A Study on Frequency of Development of Polycystic Ovarian Syndrome Among Undergraduate Doctor of Physical Therapy Students
}

\author{
SAIMA RIAZ1 ${ }^{1}$, ANFAL ZAHRA ${ }^{2}$, ZEEST HASHMI ${ }^{3}$, SAJID RASHID $^{4}$, REHAN RAMZAN KHAN ${ }^{5}$, AMBER SHABBIR $^{6}$ \\ ${ }^{1}$ Assistant Professor, Riphah College of Rehabilitation and Allied Health Sciences, Riphah International University, Lahore, Pakistan. \\ ${ }^{2}$ Internee Physiotherapist, Riphah College of Rehabilitation and Allied Health Sciences, Riphah International University, Lahore, Pakistan. \\ ${ }^{3}$ Senior Lecturer, Riphah College of Rehabilitation and Allied Health Sciences, Riphah International University, Lahore, Pakistan. \\ ${ }^{4}$ Professor, Multan College of Physiotherapy, Multan Medical \& Dental College, Multan, Pakistan. \\ ${ }^{5}$ Assistant Professor, Multan College of Physiotherapy, Multan Medical \& Dental College, Multan, Pakistan. \\ ${ }^{6}$ Senior Lecturer, Dakson Institute of Health Scinces, Islamabad, Pakistan. \\ Correspondence to: Dr. Saima Riaz, MS-OMPT, PhD*, Email: saima.zahid@riphah.edu.pk, Cell: 0333-4349520
}

\begin{abstract}
Objective: To find out the frequency of development of polycystic ovarian syndrome among undergraduate Doctor of Physical Therapy students.

Methodos: It was a cross-sectional study. Data was collected from Riphah International University, Lahore. The study was conducted on 350 undergraduate doctor of physical therapy students. Non-probability convenience sampling technique was used. Sample collection was done based on pre defined inclusion and exclusion criteria. Polycystic ovarian syndrome self-assessment form based on WHO guidelines was used. Collected data was entered and analyzed on SPSS version 21

Results: The target population of research comprises of 350 participants. The mean age of students was 21.28 \pm 2.35 . Out of which $35.71 \%$ were hostilities and $64.29 \%$ were day scholars. Among hostellites $49.6 \%(n=62)$ were at risk of unpredictable PCOS, 39.2\% $(n=49)$ were at chance of getting PCOS and $11.2 \%(n=14)$ were at high risk to get PCOS. In day scholars $52.9 \%(n=119)$ were at risk of unpredictable PCOS, $39.6 \%(n=89)$ were at chance of getting PCOS and $7.6 \%(n=17)$ were at high risk of PCOS

Conclusion: The current study concludes that polycystic ovarian syndrome (PCOS) is more prevalent among female adolescents which may cause obstacles in their lifestyle ultimately leading to infertility. This negative effect is due to the unhealthy stressful lifestyle of teenager female adolescents. The unpredictable polycystic ovarian syndrome is more frequent in day scholars than hostilities among Doctor of Physical Therapy Students.

Keywords: Polycystic ovary syndrome, Hyperinsulinism, Hirsutism
\end{abstract}

\section{INTRODUCTION}

Polycystic Ovarian Syndrome (PCOS) is characterized by multiple small ovarian cysts, obesity, hypertension, diabetes, insulin resistance, and Hirsutism. There are various elements of the polycystic ovarian syndrome; however, three main dominant factors are the irregular menstruation cycle which means ovaries do not release eggs regularly, excessive levels of the male hormone, which causes growth of excessive body and facial hairs and ovaries of a young female enlarge pertaining follicles (1). Several factors contribute towards polycystic ovarian syndrome that encompasses the family history of the polycystic ovarian syndrome, obesity and unhealthy dietary habits $(2,3)$. It is considered a common endocrine disorder that affects reproductive aged women (4). Studies revealed polycystic ovarian syndrome contributes towards poor quality of life with the symptoms of depression provoking suicidal thoughts associated with body image disturbance and low self esteem affecting sleeping patterns $(5,6)$.

This is a typical heterogeneous disorder in teens or early young age categorized in anovulation (amenorrhoea, oligomenorrhoea, irregular cycles) combined with indications of increased androgen level causing hirsutism, acne and alopecia (3,7-9). The characteristic biochemical abnormalities are above the expected level of serum androgen concentrations. Significantly, polycystic ovarian syndrome (PCOS) are related to metabolic abnormalities and mainly these are insulin resistance and hyperinsulinemia which potentially carries a high risk of developing type 2 diabetes at a later age $(10,11)$. Polycystic ovarian syndrome (PCOS) is highly usual in obese girls however it varies among each centile. BMI and overweight series, Functional ovarian hyperandrogenism $(\mathrm{FOH})$ is usually the cause of androgen excess (12-14).

Although the interrelationships of complex metabolic changes between polycystic ovarian syndrome (PCOS) and obesity have yet to be understood fully. It has high significance to detect obese adolescents giving the severe consequences of the polycystic ovarian syndrome on women's reproductive, mental and cardiovascular health because future adverse effects on their health can be avoided by counselling and intervention in an early stage of the metabolic disturbances $(7,15)$. Polycystic ovarian syndrome has a strong association with insulin resistance and tends to have strong predisposition for developing type 2 diabetes, hypertension, and cardiovascular diseases. Ovarian cysts lead to hormonal imbalance. key risk factors cover obesity, sedentary lifestyle and family history of polycystic ovarian syndrome during the pubertal transition. $(16,17)$

The current study assessed the polycystic ovarian syndrome frequency among undergraduate doctor of physical therapy students. Very few published studies had been found before in Pakistan to explore this issue among undergraduate physical therapy students. 


\section{METHODS}

It was a cross-sectional study conducted on the undergraduate Doctor of Physical Therapy Students studying at Riphah International University Lahore after obtaining thical approval. Non-probability convenience sampling technique was adopted for data collection. Sample collection was done based on inclusion and exclusion criteria. Inclusion criteria pertain to all female undergraduate physical therapy students whereas exclusion criteria pertain to students who are not willing to participate and medical students other than physical therapy. The sample size consists of 350 female students aged between 18-28 years. The data collection tool was self assessment questionnaire consisted of questions pertaining to Polycystic ovarian syndrome based on WHO Guidelines to assess the frequency of the polycystic ovarian syndrome and demographics (2). The data collected was kept entirely confidential. The data was entered and analysed by using SPSS version 21 .

\section{RESULTS}

The mean age of students was $21.28 \pm 2.35$. Participants were having a mean weight of $55.95 \pm 10.54$. The mean height of the participants was $1.50 \pm 3.13$

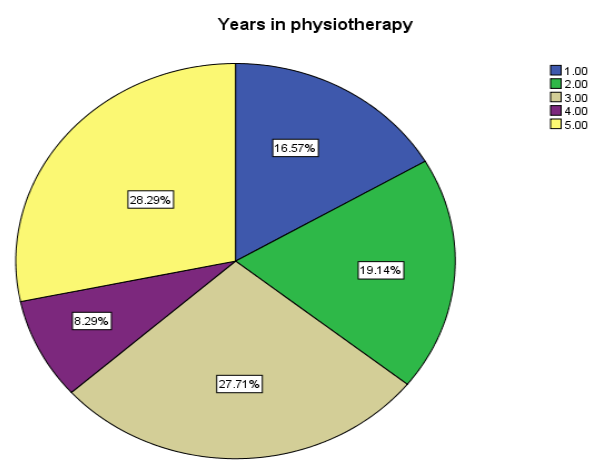

Figure-I shows that the students participating in the study were 350 out of which $16.6 \%(n=58)$ were from 1 st year of physiotherapy, $19.1 \%(n=67)$ were from 2 nd year of physiotherapy, $27.7 \% \quad(n=97)$ were from 3rd year of physiotherapy, $8.3 \%(n=29)$ years were from 4th year of physiotherapy and $28.3 \%(n=99)$ were from 5 th year of physiotherapy. The total number of students who participated in this study was 350, out of which $35.7 \%$ $(n=125)$ were Hostelities and $64.3 \%(n=225)$ were day scholars.

Table-I: PCO'S Risk Score

\begin{tabular}{|l|l|l|}
\hline PCO'S RISK & $\mathrm{N}$ & $\%$ \\
\hline Unpredictable PCO'S & 181 & 51.7 \\
\hline Chance of getting PCO'S & 138 & 39.4 \\
\hline High risk to get PCO'S & 31 & 8.9 \\
\hline
\end{tabular}

Table-I shows that total 350 students participated in this study, out of which $51.7 \% \quad(n=181)$ were at risk of unpredictable PCOS, 39.4\% $(n=138)$ were at chance of getting PCOS, and $8.9 \%(n=31)$ were at high risk to get PCOS.
Table -II: PCOS Risk Score In Hostellites And Day Scholars

\begin{tabular}{|l|l|l|l|}
\hline $\begin{array}{l}\text { Residential } \\
\text { Status }\end{array}$ & $\begin{array}{l}\text { Unpredictable } \\
\text { PCO'S } \\
\mathrm{N}(\%)\end{array}$ & $\begin{array}{l}\text { Chances of } \\
\text { getting PCO'S } \\
\mathrm{N}(\%)\end{array}$ & $\begin{array}{l}\text { High Risk of } \\
\text { PCO'S } \\
\mathrm{N}(\%)\end{array}$ \\
\hline Hostellites & $62(49.6 \%)$ & $49(39.2 \%)$ & $14(11.2 \%)$ \\
\hline Day Scholars & $119(52.9 \%)$ & $89(39.6 \%)$ & $17(7.6 \%)$ \\
\hline
\end{tabular}

Table 2 shows that the total number of students who participated in this study was 350 Out of which $35.71 \%$ were hostilities and $64.29 \%$ were day scholars. $49.6 \% \quad(n=62)$ were at risk of unpredictable PCOS $39.2 \%(n=49)$ were at chance of getting PCOS $11.2 \%(n=14)$ were at high risk to get PCOS.in day scholars $52.9 \%(n=119)$ were at risk of unpredictable PCOS $39.6 \% \quad(n=89)$ were at chance of getting PCOS and $7.6 \%(n=17)$ were at high risk of PCOS.

\section{DISCUSSION}

In this research, 350 individuals participated wilfully. The research participants were young females between the ages of (18 -28 years old) of Doctor of Physical Therapy Day scholars and hostilities students regardless of their demographic background. Most of the population was inclined towards obesity. The study examined that in hostilities students the chance of unpredictable polycystic ovarian syndrome is $49.6 \%$ whereas the chance of getting polycystic ovarian syndrome is $39.2 \%$. The frequency of participants with high risk of developing polycystic ovarian syndrome was $11.2 \%$. On the other hand, in day scholar's population study showed that unpredictable polycystic ovarian syndrome among undergraduate students was $52.9 \%$. However $39.6 \%$ of females had a chance of getting polycystic ovarian syndrome while students on high-risk polycystic ovarian syndrome were $7.6 \%$. According to this research study $52.9 \%$ population presented with unpredictable polycystic ovarian syndrome, which is a significant number that cannot be ignored.

In comparison to Mahesh Gupta et al. in 2018 research work on polycystic ovarian syndrome among young women in Bhopal, Central India, this study found significant relevance showing the results in all those girls who are with higher BMI (16).

Aneesa Thannickal et al., conducted a study in 2020 and found that females with the polycystic ovarian syndrome had an increased tendency to have diabetes and hypertension. Furthermore, her study showed an impact on sleeping patterns and disordered eating habits (18). Current study also concludes that lifestyle and eating habits are linked with the risk of developing PCOs in young girls. This study also shows that young girls with polycystic ovarian syndrome (PCOS) had an obesity craving for carbohydrates and irregular cycles.

Dr. Beena Joshi et al. in 2014 conducted a crosssectional survey on the polycystic ovarian syndrome to assess the prevalence of polycystic ovarian syndrome (PCOS) among 778 adolescents aged 15-25 years and concluded that polycystic ovarian (PCOS) is an intervening disorder in young females and early screening and intervention may provide beneficial results in promoting healthy lifestyle (12). The results of current study are in agreement with this study.

M. Manasa Rekha conducted a study on identifying risk factors in developing Poly Cystic Ovarian Syndrome among teenagers and minimizing them by Lifestyle 
Modifications through Advanced Patient Counselling (19). They found that 51.13 percent of participants had the chance of getting PCOS, whereas the current study showed 39.4 percent were at the chance of getting polycystic ovarian syndrome. However, in this comparison, there may be demographic, racial, and lifestyle factors were ignored. In M. Manasa Rekha study 159 participants with scoring $>10$ with percentile 30.01 were with a high chance for getting polycystic ovarian syndrome (PCOS), 100 with scoring $<5$ with percentile 18.86 were unpredictable to the polycystic ovarian syndrome (19). Whereas in comparison to this study, indicated out of 350 participants, 8.9 percent were at high chance of getting polycystic ovarian syndrome (PCOS) and 51.7 percent were unpredictable to polycystic ovarian syndrome (PCOS).

\section{CONCLUSION}

The current study concludes that polycystic ovarian syndrome (PCOS) is more prevalent among female adolescents which may cause obstacles in their lifestyle ultimately leading to infertility. This negative effect is due to the unhealthy stressful lifestyle of teenager female adolescents. The unpredictable polycystic ovarian syndrome is more frequent in day scholars than hostilities among Doctor of Physical Therapy Students.

\section{REFERENCES}

1. Attlee A, Nusralla A, Eqbal R, Said H, Hashim M, Obaid RS. Polycystic ovary syndrome in university students: occurrence and associated factors. International journal of fertility \& sterility. 2014;8(3):261.

2. Begum G, Shariff A, Ayman G, Mohammad B, Housam R, Khaled N. Assessment of risk factors for development of polycystic ovarian syndrome. diabetes. 2017;1:2.

3. Zawadzski J. Diagnostic criteria for polycystic ovary syndrome: towards a rational approach. Polycystic ovary syndrome. 1992:39-50.

4. Mani H, Levy MJ, Davies MJ, Morris DH, Gray LJ, Bankart J, et al. Diabetes and cardiovascular events in women with polycystic ovary syndrome: a 20 -year retrospective cohort study. Clinical endocrinology. 2013;78(6):926-34.

5. Kutenaee MA, Amirjani S, Asemi Z, Taghavi S-A, Allan H, Kamalnadian S-N, et al. The impact of depression, selfesteem, and body image on sleep quality in patients with PCOS: a cross-sectional study. Sleep and Breathing. 2019:1-8.

6. Chaudhari AP, Mazumdar K, Mehta PD. Anxiety, depression, and quality of life in women with polycystic ovarian syndrome. Indian journal of psychological medicine. 2018;40(3):239-46.

7. Witchel SF, Oberfield SE, Peña AS. Polycystic ovary syndrome: pathophysiology, presentation, and treatment with emphasis on adolescent girls. Journal of the Endocrine Society. 2019;3(8):1545-73.

8. Hohl A, Ronsoni MF, Oliveira Md. Hirsutism: diagnosis and treatment. Arquivos Brasileiros de Endocrinologia \& Metabologia. 2014;58:97-107.

9. Rebar R. Evaluation of amenorrhea, anovulation, and abnormal bleeding. 2015.

10. Franks S. Polycystic ovary syndrome. New England Journal of Medicine. 1995;333(13):853-61.

11. Rosenfield RL, Ehrmann DA. The pathogenesis of polycystic ovary syndrome (PCOS): the hypothesis of PCOS as functional ovarian hyperandrogenism revisited. Endocrine reviews. 2016;37(5):467-520.

12. Joshi B, Mukherjee $S$, Patil A, Purandare A, Chauhan S, Vaidya R. A cross-sectional study of polycystic ovarian syndrome among adolescent and young girls in Mumbai, India. Indian journal of endocrinology and metabolism. 2014;18(3):317.

13. Rosenfield RL, Bordini B. Evidence that obesity and androgens have independent and opposing effects on gonadotropin production from puberty to maturity. Brain research. 2010;1364:186-97.

14. Palomba S, Santagni S, Falbo A, La Sala GB. Complications and challenges associated with polycystic ovary syndrome: current perspectives. International journal of women's health. 2015;7:745.

15. Vilmann LS, Thisted E, Baker JL, Holm J-C. Development of obesity and polycystic ovary syndrome in adolescents. Hormone research in paediatrics. 2012;78(5-6):269-78.

16. Gupta M, Singh D, Toppo M, Priya A, Sethia S, Gupta P. A cross sectional study of polycystic ovarian syndrome among young women in Bhopal, Central India. Int $\mathrm{J}$ Community Med Public Health. 2018;5(1):95-100.

17. Allahbadia GN, Merchant R. Polycystic ovary syndrome and impact on health. Middle East Fertility Society Journal. 2011;16(1):19-37.

18. Thannickal A, Brutocao C, Alsawas M, Morrow A, Zaiem F, Murad $\mathrm{MH}$, et al. Eating, sleeping and sexual function disorders in women with polycystic ovary syndrome (PCOS): A systematic review and meta-analysis. Clinical endocrinology. 2020;92(4):338-49.

19. Rekha MM, Manasa M, Reddy C. A Study on identification of risk factors in developing Poly Cystic Ovarian Syndrome among teenagers and minimizing them by Life Style Modifications through Advanced Patient Counselling by Doctor of Pharmacy. PharmaTutor. 2018;6(1):72-8. 